\title{
Investigation of Foot Pressure Distribution in Asymptomatic Individuals with Mild Hallux Valgus
}

\author{
(i) Serkan TAŞ
}

Toros University Faculty of Health Sciences, Department of Physiotherapy and Rehabilitation, Mersin, Turkey

\begin{abstract}
Objective: The purpose of the present study was to investigate plantar pressure distribution during static standing in asymptomatic individuals with mild hallux valgus and to compare the results with healthy controls group.

Methods: This study included a total of 25 asymptomatic individuals with hallux valgus (22 females, 3 males) between the ages of 19 and 51 years. The controls group comprised of 28 health individuals without hallux valgus ( 25 females, 3 males) between the ages of $20-51$ years. Static plantar pressure distribution and force measurement during static standing in individuals were assessed using Tecscan MatScan System (Tekscan, Inc., South Boston, Massachusetts, USA).

Results: It was found that both groups have similar maximum pressure $(\mathrm{p}=0.669)$, mean pressure $(\mathrm{p}=0.950)$, heel maximum force $(\mathrm{p}=0.660)$, midfoot maximum force $(\mathrm{p}=0.894)$, metatarsal maximum force $(\mathrm{p}=0.824)$, contact area $(\mathrm{p}=0.695)$, force-time integral $(0.498)$, pressure-time integral $(\mathrm{p}=0.769)$ and center of force $(\mathrm{p}=0.178)$.

Conclusion: The results of the present study show that asymptomatic individuals with or without mild hallux valgus have similar plantar pressure distribution. These results suggest that plantar pressure distribution do not affect the development of hallux valgus or changes of hallux position do not change plantar pressure distribution in asymptomatic individuals with mild hallux valgus.
\end{abstract}

Keywords: Hallux valgus, plantar pressures, orthopedics, foot

\section{Introduction}

Hallux valgus is a foot deformity characterized by the lateral deviation of the big toe that accompanies the medial deviation of the first metatarsal bone (1). Hallux valgus, one of the most common foot deformities in the adult population, is associated with many demographics and biomechanical abnormalities such as age, female sex, use of narrow-nosed shoes, short achilles tendon, ligament laxity, and increased pronation in the back foot (2-5). Hallux valgus deformity is often accompanied by callus formation in the big toe, subluxation of the first metatarsophalangeal joint and pain (6). These symptoms that accompany hallux valgus can cause a significant reduction in the person's quality of life $(7,8)$.

Plantar foot pressure distribution measurement provides important information about foot and ankle function during walking or other functional activities (9). Plantar pressure distribution is frequently used in the evaluation and treatment plan of individuals with hallux valgus as well as neurological and musculoskeletal diseases associated with foot disorders (9-13). There is a correlation between the presence of hallux valgus and the reduction in the medial longitudinal arch (14). It is thought that changes in the position of the big toe as well as a decrease in the medial longitudinal arch in individuals with hallux valgus 
may cause changes in plantar pressure distribution. When the literature is examined, it is observed that changes in plantar pressure distribution in individuals with hallux valgus are the subject of many studies (13,15-20). However, when these studies are examined, it is observed that the plantar pressure distribution of elderly individuals with severe hallux valgus was investigated during walking $(13,16-18,20)$ and/or the presence of pain was not questioned in the evaluated individuals (13,15-18). Changes in plantar pressure distribution in asymptomatic individuals with young and mild hallux valgus were not the subject of any studies. Therefore, the aim of this study was to investigate plantar pressure distribution in static standing posture in asymptomatic individuals with mild hallux valgus and compare it with healthy individuals without hallux valgus. The hypothesis of this study is that the plantar pressure distribution of individuals with mild hallux valgus will differ from the control group.

\section{Methods}

\section{Power Analysis}

Power analysis was conducted to determine the number of cases in this study. In order to perform this study with $80 \%$ power and 5\% error margin, it was found that in case the peak pressure value of the control group is $140 \mathrm{kPa}$ and the standard deviation is $40 \mathrm{kPa}$, a minimum of 15 individuals in each group are needed to detect a deviation of $40 \mathrm{kPa}$ at the peak pressure $(15,17)$.

\section{Subjects}

This study was conducted with the participation of 53 individuals, including 6 males and 47 females in the age range of 19-51 years. The hallux valgus group consisted of a total of 25 individuals, including 3 males and 22 females with asymptomatic hallux valgus who did not suffer any pain associated with hallux valgus in the age range of 19-51 years. The control group was made up of 28 individuals, including 3 males and 25 females without hallux valgus in the age range of 20-51 years. Assessments within the scope of this study were performed in the Toros University. Individuals with asymptomatic hallux valgus and the control group were formed from the relatives of the researcher. In the study, patients with plantar fasciitis, foot injuries such as achilles tendinopathy, rheumatic diseases such as rheumatoid arthritis or osteoarthritis, history of major trauma or surgery involving the foot and/or lower extremities and significant postural disorder involving the foot and/or lower extremities were not included. In order to carry out this study, the necessary approval was obtained from the Toros University Non-interventional Ethics Committee (decision No: 2018-03/05). Individuals who read and approved the informed consent form were included in this study.

\section{Hallux Valgus Assessment}

The individuals 'big toe position was evaluated using the Manchester Scale, which was defined as valid and reliable in clinical evaluation $(21,22)$. The scale consists of 4 phases. Stage 0 shows that there is no deformity and it is the normal big toe position. Stage 3 indicates the presence of severe hallux valgus.
Only asymptomatic individuals with Stage 1 hallux valgus were included in the study. The assessment of the position of the big toe according to the Manchester scale of individuals was done by a physiotherapist with 27 years of experience in foot health and diseases. Also the hallux angle of the toe of individuals was measured using goniometric measurement. Goniometric measurements were made while individuals were in a standing upright position. While the first metatarsophalangeal joint was designated as the pivot point of the goniometer, a lever of the goniometer was placed on the metatarsal bone and its other arm was placed as parellel to the proximal phalanx. The hallux angle was recorded in degrees.

\section{Measurements of Foot Pressure Distribution}

Measurements of static foot plantar pressure distribution of the subjects were evaluated using the Tecscan MatScan systems, which were reported to be reliable and valid (Texcan, Inc., South Boston, Massachusetts, USA) (23,24). Measurements were made with bare feet, standing in a comfortable upright position for 30 seconds. The peak pressure $(\mathrm{kPa})$ average pressure $(\mathrm{kPa})$, peak force of the heel (Ibs), mid-foot peak force (Ibs), forefoot peak force (Ibs), contact area $\left(\mathrm{cm}^{2}\right)$, the force-time integral (Ibs*s), pressure-time integral $\left(\mathrm{kPa}^{*} \mathrm{~s}\right)$ and the change in the center of force $(\mathrm{cm})$ were recorded (Figure 1).

\section{Statistical Analysis}

Statistical analysis was performed using SPSS for Windowsversion 22 software. The compatibility of variables with normal distribution was examined using visual (histogram and probability graphs) and analytical methods (Kolmogorov-Smirrov/ShapiroWilk tests). Demographic data and the parameters of the pedographic analysis results were evaluated using descriptive analyses and were presented using the median and interquartile range. Since it was determined that the parameters evaluated did not show normal distribution, these parameters were compared between the groups using the Mann-Whitney U test. Cases where $\mathrm{p}$ value is less than 0.05 were evaluated as statistically significant results.

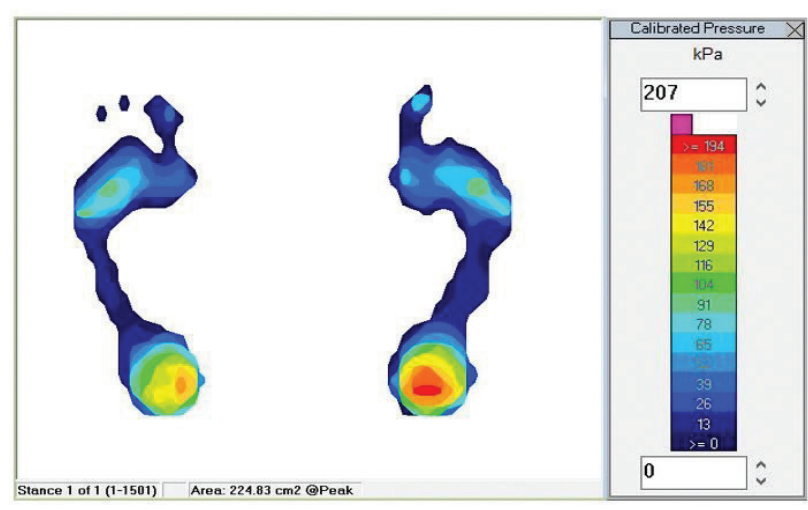

Figure 1. Plantar pressure distribution map obtained from pedographic analysis 


\section{Results}

Age ( $\mathrm{p}=0.858)$, height $(\mathrm{p}=0.412)$, weight $(\mathrm{p}=0.762)$ and body mass index $(\mathrm{p}=0.581)$ of both groups included in the study were found to be similar (Table 1).

It was found that the hallux angle of individuals with hallux valgus increased compared with the control group $(p<0.001)$. Plantar pressure distribution analysis results showed that the peak pressure $(p=0.669)$, average pressure $(p=0.950)$, heel peak force $(p=0.660)$, mid-foot peak force $(p=0.894)$, forefoot peak force $(p=0.824)$, contact area $(p=0.695)$, the force-time integral $(p=0.498)$, pressure-time integral $(p=0.769)$, and changes in centre of force $(p=0.178)$ parameters were similar in both groups (Table 2).

\section{Discussion}

This study was planned to investigate plantar pressure distribution in asymptomatic individuals with mild halllux valgus. The results showed that, unlike the hypothesis of the study, the results of plantar pressure analysis of individuals with and without hallux valgus were similar. Similar to the results obtained, Iliou et al. (15) showed that average of the pressures in the metatarsal region was similar in individuals with mild hallux valgus and without hallux valgus, and that the average pressure significantly increased at first and second metatarsal heads in individuals with moderate or severe hallux valgus. Unlike the results obtained, Hida et al.
(13) reported decreased peak force, contact area, contact time and force-time integral compared and increased forefoot peak pressure and force values in individuals with moderate and severe hallux valgus compared with the control group during walking. Mickle et al. (16) reported increased peak pressure, pressure-time integral and peak pressure in the first metatarsal region in the aged population with hallux valgus. Galica et al. (17) reported increased peak pressure in the front foot and abnormal pressure distribution in the rear foot in the geriatric population with hallux valgus. Wen et al. (20) reported an overload in the first and second metatarsal bones, a decrease in the amount of pressure around the hallux and a collapse in the medial longitudinal arch. As seen here, the studies in the literature differ markedly from the results we obtained. There may be several major reasons for this. First, a significant portion of these studies were conducted on individuals of advanced age with significant hallux valgus. In addition, individuals with pain were included in these studies or the pain of individuals was not questioned. In addition, in all studies, foot pressure distribution of individuals with hallux valgus was evaluated during walking and foot pressure distribution in static condition was not evaluated.

In this study, the foot pressure distribution of individuals was evaluated in the static state and it was found that the change in the foot pressure center of individuals with hallux valgus was similar to that of the control group. These results indicate that there is no loss of postural stability in asymptomatic individuals

Table 1. Median values of demographics of groups (interquartile range/ $\% 25-\% 75$ percentile)

\begin{tabular}{|c|c|c|c|}
\hline Parameters & Control Group & HV Group & $p$ \\
\hline Age (year) & $35.5(25.5-42.5)$ & $31.0(25.0-46.0)$ & 0.448 \\
\hline Gender (F/M) & $25 / 3$ & $22 / 3$ & - \\
\hline Height (m) & $1.62(1.58-1.65)$ & $1.62(1.57-1.68)$ & 0.140 \\
\hline Weight (kg) & $63.5(55.8-70.1)$ & $62.2(55.4-67.2)$ & 0.670 \\
\hline BMI $\left(\mathrm{kg} / \mathrm{m}^{2}\right)$ & $23.8(21.9-26.1)$ & $23.4(21.4-25.6)$ & 0.408 \\
\hline Hallux angle $\left({ }^{\circ}\right)$ & $12(10-13)$ & $21(19-22)$ & $<0.001$ \\
\hline
\end{tabular}

BMI: Body mass index, HV: Hallux Valgus, F: Female, M: Male

Table 2. Median values of plantar pressure analysis results of groups (interquartile range/ \%25-\%75 percentile)

\section{Parameters}

Peak pressure $(\mathrm{kPa})$

Average pressure ( $\mathrm{kPa})$

Heel peak force (Ibs)

Mid-foot peak force (Ibs)

Front foot peak force (Ibs)

Contact area $\left(\mathrm{cm}^{2}\right)$

Force-time integral (lbs*s)

Pressure-time integral ( $\left.\mathrm{kPa}{ }^{*} \mathrm{~s}\right)$

Change in centre of force $(\mathrm{cm})$

kPa: Kilopascal; Ibs: Pound

\section{Control Group}

132 (102-164)

108 (80-139)

$30.9(27.7-38.9)$

$20.4(16.2-28.6)$

$32.7(27.1-37.4)$

91.8 (77.5-99.2)

359.1 (325.7-407.4)

$187.0(165.5-198.0)$

$6.10(5.2-8.2)$

\begin{tabular}{ll} 
HV Group & $P$ \\
\hline $124(103-162)$ & 0.238 \\
$108(81-128)$ & 0.568 \\
$31.0(25.0-42.6)$ & 0.566 \\
$21.4(15.6-28.8)$ & 0.254 \\
$30.2(26.4-39.0)$ & 0.910 \\
$91.5(84.0-98.0)$ & 0.827 \\
$385.1(324.1-445.9)$ & 0.930 \\
$181.0(170.0-206.0)$ & 0.665 \\
$7.0(6.1-8.4)$ & 0.121 \\
\hline
\end{tabular}


with mild hallux valgus. Similar to the results we obtained, Kavlak (25) found that hallux valgus did not affect static and dynamic balance in the geriatric population. Hurn et al. (26) examined the relationship between the balance performance on one leg and the severity of hallux valgus in a study and found that there was no loss of balance in individuals with mild and moderate hallux valgus and that the amount of mediolateral oscillation increased in individuals with severe hallux valgus.

This study had some limitations. Firstly, this study was conducted only in young and asymptomatic individuals with mild hallux valgus. If geriatric individuals and individuals with different severity of hallux valgus were included in the study, differences in plantar pressure distribution could be revealed in line with the progression of hallux valgus. Second, the pedographic analyses performed in this study were performed by a non-blind evaluator, but we believe that the measurement using standard methods minimalized possible biases. Finally, the majority of the individuals evaluated in this study consisted of women. However, similar gender distribution of individuals with hallux valgus in our study and in studies in the literature suggests that this would not result in a significant change in results.

\section{Conclusion}

As a result, it was found that pressure and force distributions of asymptomatic individuals with mild hallux valgus and the control group without hallux valgus were similar. The results suggest that in individuals with asymptomatic mild hallux valgus, hallux valgus development does not affect plantar pressure distribution, or that plantar pressure distribution is not associated with hallux valgus development. Furthermore, similar changes in the center of force in asymptomatic individuals with mild hallux valgus and in the control group indicate that postural control is not affected in asymptomatic individuals with mild hallux valgus.

\section{Ethics}

Ethics Committee Approval: Toros University NonInterventional Ethics Committee (decision No: 2018-03/05).

Informed Consent: Individuals who read and approved the informed consent form were included in this study.

Peer-review: Internally peer-reviewed.

Financial Disclosure: The authors declared that this study received no financial support.

\section{References}

1. Glasoe WM, Nuckley DJ, Ludewig PM. Hallux valgus and the first metatarsal arch segment: a theoretical biomechanical perspective. Phys Ther 2010;90:110-20.

2. Hagedorn TJ, Dufour AB, Riskowski JL, Hillstrom HJ, Menz HB, Casey VA, et al. Foot disorders, foot posture, and foot function: the Framingham foot study. PLoS One 2013;8:e74364.

3. Choi GW, Kim HJ, Kim TW, Lee JW, Park SB, Kim JK. Sex-related differences in outcomes after hallux valgus surgery. Yonsei Med J 2015;56:466-73.
4. Barouk LS. The effect of gastrocnemius tightness on the pathogenesis of juvenile hallux valgus: a preliminary study. Foot Ankle Clin 2014;19:807-22.

5. Xiang L, Mei Q, Fernandez J, Gu Y. Minimalist shoes running intervention can alter the plantar loading distribution and deformation of hallux valgus: A pilot study. Gait Posture 2018;65:6571.

6. Suzuki J, Tanaka Y, Takaoka T, Kadono K, Takakura Y. Axial radiographic evaluation in hallux valgus: evaluation of the transverse arch in the forefoot. J Orthop Sci 2004;9:446-51.

7. Lopez DL, Callejo Gonzalez L, Losa Iglesias ME Canosa JL, Sanz DR, Lobo CC, et al. Quality of Life Impact Related to Foot Health in a Sample of Older People with Hallux Valgus. Aging Dis 2016;7:4552.

8. Lopez DL, Fernandez JM, Iglesias ME Castro CÁ, Lobo CC, Galván $\mathrm{JR}$, et al. Influence of depression in a sample of people with hallux valgus. Int J Ment Health Nurs 2016;25:574-8.

9. Orlin MN, McPoil TG. Plantar pressure assessment. Phys Ther 2000;80:399-409.

10. Ünver B, Bek N. Tabanlik kullaniminin plantar temas alanlari ve basinç dağilimina etkisi. Turk J Physiother Rehabil 2014;2:1-7.

11. Ma CZ, Zheng YP, Lee WC. Changes in gait and plantar foot loading upon using vibrotactile wearable biofeedback system in patients with stroke. Top Stroke Rehabil 2018;25:20-7.

12. Fang F, Wang YF, Gu MY Chen H, Wang DM, Xiao K, et al. Pedobarography - a novel screening tool for diabetic peripheral neuropathy? Eur Rev Med Pharmacol Sci 2013;17:3206-12.

13. Hida T, Okuda R, Yasuda T, Jotoku T, Shima H, Neo M. Comparison of plantar pressure distribution in patients with hallux valgus and healthy matched controls. J Orthop Sci 2017;22:1054-9.

14. Blackwood S, Gossett L. Hallux Valgus/Medial Column Instability and Their Relationship with Posterior Tibial Tendon Dysfunction. Foot Ankle Clin 2018;23:297-313.

15. Iliou K, Paraskevas G, Kanavaros P, Gekas C, Barbouti A, Kitsoulis P. Relationship between pedographic analysis and the Manchester scale in hallux valgus. Acta Orthop Traumatol Turc 2015;49:75-9.

16. Mickle KJ, Munro BJ, Lord SR, Menz HB, Steele JR. Gait, balance and plantar pressures in older people with toe deformities. Gait Posture 2011;34:347-51.

17. Galica AM, Hagedorn TJ, Dufour AB, Riskowski JL, Hillstrom HJ, Casey VA, et al. Hallux valgus and plantar pressure loading: the Framingham foot study. J Foot Ankle Res 2013;6:42.

18. Koller U, Willegger M, Windhager R, Wanivenhaus A, Trnka HJ, Schuh R. Plantar pressure characteristics in hallux valgus feet. J Orthop Res 2014;32:1688-93.

19. Nakajima K, Kobayashi Y, Tada M, Mochimaru M. Evaluation of plantar pressures in people with hallux valgus using principal component analysis. Technol Health Care 2018;26:667-74.

20. Wen J, Ding Q, Yu Z, Sun W, Wang Q, Wei K. Adaptive changes of foot pressure in hallux valgus patients. Gait Posture 2012;36:344-9. 
21. Menz HB, Fotoohabadi MR, Wee E, Spink MJ. Validity of selfassessment of hallux valgus using the Manchester scale. BMC Musculoskelet Disord 2010;11:215.

22. Garrow AP, Papageorgiou A, Silman AJ, Thomas E, Jayson MI, Macfarlane GJ. The grading of hallux valgus: the Manchester Scale. J Am Podiatr Med 2001;91:74-8.

23. Goetschius J, Feger MA, Hertel J, Hart JM. Validating Center-ofPressure Balance Measurements Using the MatScan ${ }^{\circledast}$ Pressure Mat. J Sport Rehabil 2018;27.
24. Wettenschwiler PD, Stämpfli R, Lorenzetti S, Ferguson SJ, Rossi RM, Annaheim S. How reliable are pressure measurements with Tekscan sensors on the body surface of human subjects wearing load carriage systems? Int J Ind Ergon 2015;49:60-7.

25. Kavlak Y. The relation of hallux valgus severity with foot function and balance in older men. Turk J Physiother Rehabil 2015;26:93-9.

26. Hurn SE, Vicenzino B, Smith MD. Functional impairments characterizing mild, moderate, and severe hallux valgus. Arthritis Care Res (Hoboken) 2015;67:80-8. 